\title{
3D iterative reconstruction can do so much more than reduce dose
}

\author{
James A. Case, Ph.D. MASNC \\ a Cardiovascular Imaging Technologies, Kansas City, MO
}

Received Jun 20, 2019; accepted Jun 21, 2019

doi: $10.1007 / \mathrm{s} 12350-019-01827-4$

\begin{abstract}
Recent advances in software and hardware for cardiac SPECT have the potential to revolutionize nuclear cardiology. It is easy to use these technologies to maintain the status quo and lower radiation dose, despite the fact there is very little evidence that lowering patient dose in already low dose imaging protocols confers any benefit to patients. Cardiac SPECT has tremendous potential for risk stratification, molecular tracers, and high temporal resolution management of patients with electrophysiological disorders. In addition, these new reconstruction techniques can offer spatial resolution that is comparable and sometimes even superior to PET. Lastly, recent research has also held out the potential for performing absolute blood flow qualification using SPECT instrumentation. As these new technologies become available, the goal should be to make images better and improve patient care first, then optimize the dose.
\end{abstract}

Key Words: Image reconstruction $\cdot$ modalities $\bullet$ instrumentation

See related article, https://doi.org/10.10 07/s12350-019-01743-7.

\section{INTRODUCTION}

Since the discovery of the X-ray in the early 20th century, ionizing radiation has revolutionized the practice of medicine in ways that are hard to fathom. Its impact on the care of patients with heart disease, specifically using radionuclides, has meant earlier detection, better outcomes, and better patient care. All these benefits came with few contraindications or side effects. The approval of Tl-201 in 1979 allowed for a whole body radiation dose of up to $40 \mathrm{mSv}{ }^{1}$ In the $1990 \mathrm{~s}$, technetium tracers with their shorter half-lives simultaneously reduced patient dose to $18 \mathrm{mSv}$ while increasing overall image quality. ${ }^{2}$ The initial recommendations for Tc $99 \mathrm{~m}$ sestamibi was a $30 \mathrm{mCi}$ dose on the first day followed by a second $30 \mathrm{mCi}$ dose on the second day.

\footnotetext{
Reprint requests: James A. Case, Ph.D. MASNC, Cardiovascular Imaging Technologies, 4320 Wornall Road Suite 114, Kansas City, MO 64111; jcase@cvit.com

J Nucl Cardiol 2021;28:2633-7.

1071-3581/\$34.00

Copyright (C) 2019 American Society of Nuclear Cardiology.
}

This protocol was improved upon by validating a same day, low $10 \mathrm{mCi}$ resting dose followed by a higher dose, $30 \mathrm{mCi}$ dose at stress, with a corresponding dose reduction from 18.6 to $12.6 \mathrm{mSv}$. At each step along the way, image quality was improved alongside the reduction of radiation. These protocols, in conjunction with dual headed anger camera instrumentation, became the backbone of nuclear cardiology throughout the 1990s and 2000s.

More recently, professional societies and government agencies have raised concerns that given the increased number of radiological studies patients receive over their lifetime, there may be a potential risk of radiation induced carcinogenesis. ${ }^{3-5}$ This risk from diagnostic ionizing radiation is based off a linear extrapolation of radiation effects at very high radiation dosages to the low radiation exposure used in medical imaging, referred to as the linear no-threshold (LNT) model. ${ }^{6}$ Much of these data come from nuclear power plant accidents and atomic bomb survivor data. Though this is the most commonly accepted model, there is considerable evidence that radiation induced carcinogenesis does not obey this model. ${ }^{7-10}$ There is even more recent evidence suggesting DNA repair mechanisms are activated when exposed to low doses of 
radiation, decreasing cellular sensitivity to radiation. ${ }^{11,12}$ The LNT model for radiation effects in these acute exposure settings bare little similarity to the conditions in diagnostic radiology. This is not to say that nuclear cardiologists should ignore recommendations to avoid unnecessary radiation exposure, rather it is to look carefully at what is really meant by unnecessary. Specifically, when efforts to reduce radiation exposure prevent nuclear cardiology from innovating and improving image quality, it neither serves the patients nor the public.

Pretorius et al. present an excellent analysis of 3-D iterative reconstruction with resolution recovery and attenuation correction to demonstrate comparable reader accuracy at one quarter of a conventional dose. They demonstrate that global sensitivity and specificity is similar for full dose and one quarter dose myocardial perfusion imaging when data were reconstructed using OSEM with resolution recovery, scatter correction and attenuation correction. Filtered back-projection demonstrated a significant degradation in reader performance at lower injected doses. But how should we use this conclusion? One way would be is to be satisfied with the same image quality as was available in 1995 using one quarter of the dose. Another way would be to maintain the same acquisition times, and the same dosages and achieve significantly higher image quality in terms of lower noise, spatial and temporal resolution, allowing for earlier detection of disease, or detecting subtle changes in a patient's coronary disease when symptoms change.

A recent position statement from the American Heart Association and the American Society of nuclear cardiology recommended nuclear cardiology laboratories upgrade their computing capabilities to include iterative reconstruction. ${ }^{13}$ This would improve image quality substantially and reduce image artifacts at a relatively small cost to nuclear laboratories. It had also been recommended that upgrading high-resolution scanning technologies such as CZT could greatly improve overall image quality and minimize image artifacts. ${ }^{14,15}$ This statement specifically identified image quality improvement as being the greatest need in SPECT.

ALARA is often misunderstood to mean, "all radiation is bad". The Health Physics Society 2010 position paper states that for annual dose estimates of $<50 \mathrm{mSv}$ and cumulative lifetime doses of $<100 \mathrm{mSv}$, extrapolated "risk estimates should not be used". 16 This position is based on NCRP 1997 "emphasizing the inability to detect any increased health detriment" at these dose levels. ${ }^{17}$ The overstatement of radiation risk can have the unintended consequence of patients avoiding important diagnostic testing and limiting research into potentially lifesaving diagnostic advances. Some opportunities that could be missed by over emphasizing radiation risk are high temporal resolution gating, highresolution localization of disease, absolute blood flow, and the development of new tracers.

\section{HIGH TEMPORAL AND SPATIAL RESOLUTION ECG GATED SPECT}

New management strategies for heart failure and arrythmia require accurate functional information, something ECG gated SPECT obtains routinely. This is an important advantage of ECG gated SPECT has over CT and MRI-based functional imaging. Most modern SPECT cameras routinely image patients using 16 ECG gates ( $<70 \mathrm{~ms}$ per time bin). This extraordinarily high temporal resolution allows for regional assessment of the heart rhythm and wall motion abnormalities (see Figure 1). When compared to conventional $\mathrm{CT}$, with the rotation speed of 0.5 seconds, the temporal resolution can exceed $200 \mathrm{~ms}$. For MRI, ECG gating is even more challenging with the inability to scan the entire cardiac volume and acquire a 12 lead ECG necessary for stress testing. Even ${ }^{82} \mathrm{Rb}$ PET is typically limited to eight ECG time bins ( $>120 \mathrm{~ms}$ per time bin). It is possible with the advanced reconstruction techniques to achieve 32 ECG gates per study ( $<40 \mathrm{~ms}$ per time bin) with excellent image quality. This temporal resolution could prove critical in managing heart failure and EP patients as newer therapies become available.

\section{HIGH-SPATIAL RESOLUTION SPECT}

The accuracy of myocardial perfusion SPECT in detecting overall sensitivity, specificity and accuracy is well understood; however, upon closer examination SPECT performs poorly in determining the location of a coronary lesion $^{18}$ (see Figure 2). Results for myocardial perfusion PET demonstrate that with higher resolution, and scatter and attenuation correction it is possible to obtain good localization of coronary lesions. The current study examined sensitivity and specificity as global variables; however, in real world patient management, it is more important to identify specific regions of ischemia. In addition, only a small portion of patients referred for perfusion testing are truly CAD naïve. Patient management in nuclear cardiology more typically involves comparisons of subtle changes from one year to the next, patency of graphs and stents, and small changes in ejection fraction. All of these clinical endpoints benefit from high-resolution, high quality perfusion images. 


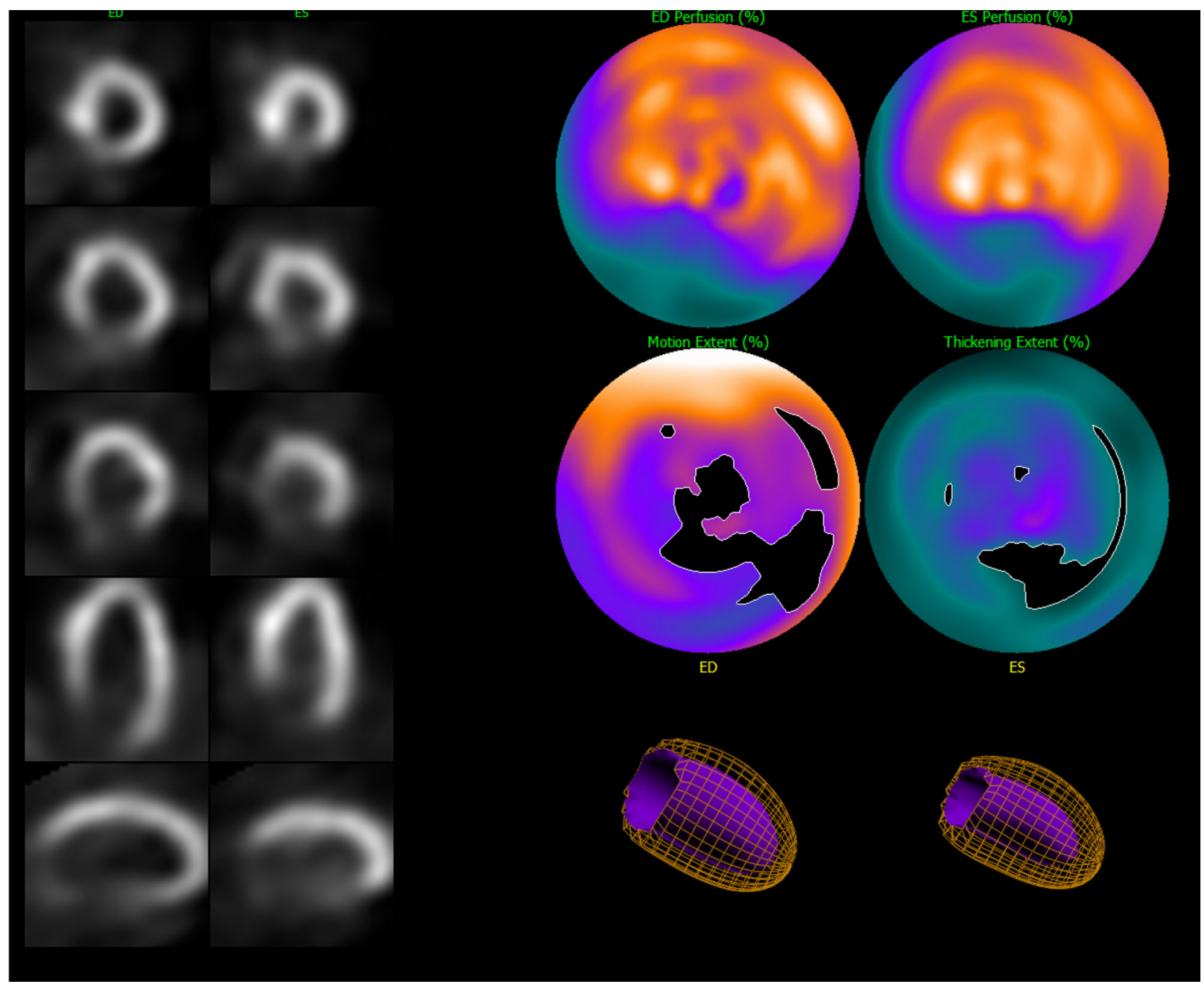

Figure 1. Very high temporal and spatial resolution SPECT greatly improves the diagnostic utility of ECG gated SPECT. Above is an iterative reconstruction, with resolution recovery image of a patient with a severe inferior and infero-lateral defect. This study was performed using $3.2 \mathrm{~mm}$ pixel size and 16 ECG gated time bins. Displayed is the end-diastolic and end systolic frames (left) and perfusion and function polar maps. In this example, ECG gated frames are reconstructed with 1/ 16 the dose of the perfusion study to achieve an incredible image of a single diastolic and systolic frame with $60 \mathrm{~ms}$ temporal resolution.

\section{SPECT FOR MYOCARDIAL BLOOD FLOW}

Myocardial perfusion PET has been revolutionized by the introduction of absolute myocardial blood flow and blood flow reserve measurements. ${ }^{19}$ More recently, there has been considerable interest in extending these advances to myocardial perfusion SPECT. ${ }^{20}$ Performing absolute myocardial blood flow with SPECT has many challenges such as lower resolution, lower temporal resolution, and more challenging radionucleotide kinetics. Despite these challenges several investigators have reported success in performing the dynamic imaging necessary for calculating myocardial blood flow. The potential benefit to patients of adding absolute blood flow and blood flow reserve information to the relative perfusion data is undeniable; however, poor signal-tonoise could compromise its utility.

\section{SPECT FOR MOLECULAR TRACERS}

Finally, adding new molecular tracers to the current stable of perfusion tracers offers the potential for further revolutionizing nuclear cardiology. Recent phase 1 data of a Tc-99m deoxyglucose molecule have opened the possibility of imaging active ischemia, and ischemic 

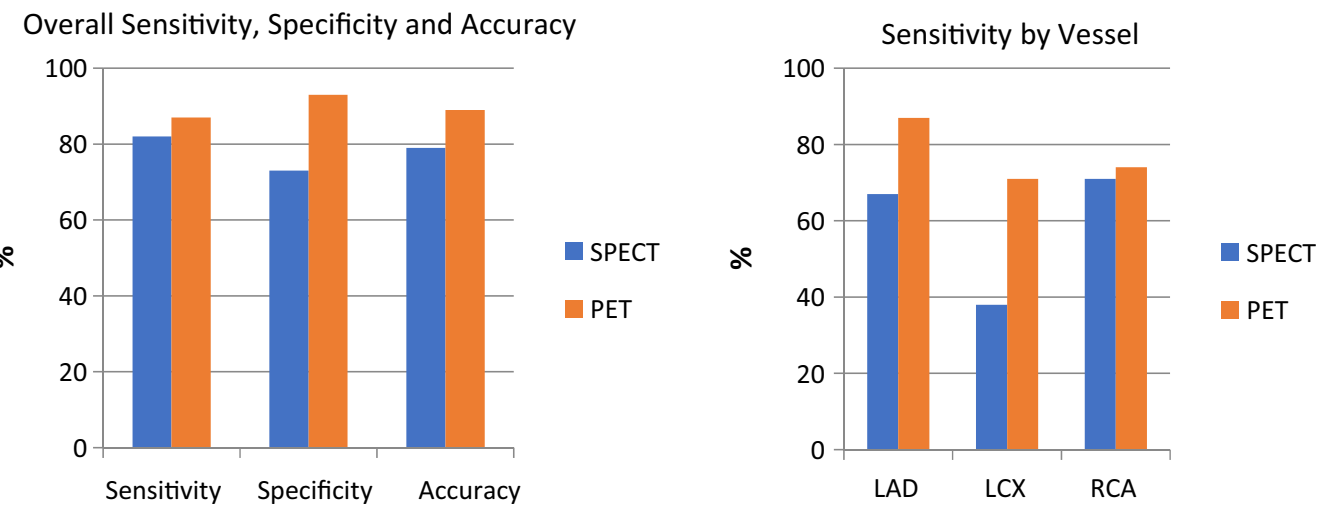

Figure 2. Overall sensitivity, specificity and accuracy of conventional FBP SPECT is similar to high cost modalities such as PET (left). By vessel, conventional FBP SPECT has a significantly lower sensitivity due to its lower resolution and artifacts (right). This inability to localize disease limits the usefulness of conventional SPECT.

memory using SPECT instrumentation. Iodine-123 has been well documented as an important radionucleotide for monoclonal antibodies and other physiologically active molecular agents, such as in MIBG and BMIPP. Despite iodine-123 obvious chemical benefits, it's long half life (12 hours) implies most imaging protocols will have a higher whole body dose when compared to technetium for PET agents. These molecular imaging agents could have immense lifesaving potential, so long as we keep in perspective the importance of imaging first and optimizing dose second.

\section{CONCLUSIONS}

Pretorius et al. have done an excellent job presenting the importance of iterative reconstruction with scatter, attenuation, and resolution compensation for improving image quality. In the simplest sense we could be content with using this conclusion to drive down radiation exposure without compromising image quality. Alternately, this conclusion could be used to spark new research into how the newer reconstruction and image processing techniques can enable the next phase of nuclear cardiology.

\section{Disclosure}

Dr. Case receive royalties for Cardiovascular Imaging Technologies for ImagenSPECT, a SPECT processing environment.

\section{References}

1. Thallius Chloride Tl-201 Injection. http://www.lantheus.com//assets/ LCR_00182_1.0_Thallium_US_PI_515039-0318.pdf. last accessed 20 June 2019.
2. International Commission on Radiological Protection, 2015. Radiation dose to patients from radiopharmaceuticals: A compendium of current information related to frequently used substances. ICRP Publication 128. Ann. ICRP 44(2S).

3. Cerqueira MD, Allman KC, Ficaro EP, Hansen CL, Nichols KJ, Thompson RC, et al. American Society for Nuclear Cardiology Information Statement: Recommendations for reducing radiation exposure in myocardial perfusion imaging. J Nucl Cardiol 2010;17:709-18.

4. Initiative to Reduce Unnecessary Radiation Exposure from Medical Imaging, U.S. Food and Drug Administration, February 2010, http://www.fda.gov/downloads/Radiation-EmittingProducts/Radia tionSafety/RadiationDoseReduction/UCM200087.pdf.

5. Image Wisely, Radiation Safety in Adult Medical Imaging, www. imagewisely.org, last 29 Apr, 2018.

6. Health Risks from Exposure to Low Levels of Ionizing Radiation: Beir Phase VII Phase 2, Committee to Assess Health Risks from Exposure to Low Levels of Ionizing Radiation, National Research Council, 2006.

7. Weber W, Zanzonico P. The controversial linear no-threshold model. J Nucl Med 2017;58:7-8.

8. Brenner DJ, Doll R, Goodhead DT, Hall EJ, Land CE, Little JB, Lubin JH, Preston DL, Preston RJ, Puskin JS, Ron E, Sachs RK, Samet JM, Setlow RB, Zaider M. Cancer risks attributable to low doses of ionizing radiation: Assessing what we really know. Proc Natl Acad Sci USA 2003;100:13761-6.

9. Nguyen PK, Lee WH, Li YF, Hong WX, Hu S, Chan C, Liang G, Nguyen I, Ong SG, Churko J, Wang J, Altman RB, Fleischmann $\mathrm{D}, \mathrm{Wu}$ JC. Assessment of the radiation effects of cardiac CT angiography using protein and genetic biomarkers. JACC Cardiovasc Imaging 2015;8:873-84.

10. Hansen CL, Hingorani R. It is time to bury the linear no threshold hypothesis. J Nucl Cardiol. 2019. https://doi.org/10.1007/s12350019-01646-7.

11. Neumaier T, Swenson J, Pham C, Polyzos A, Lo AT, Yang P, Dyball J, Asaithamby A, Chen DJ, Bissell MJ, Thalhammer S, Costes SV. Evidence for formation of DNA repair centers and dose-response nonlinearity in human cells. Proc Natl Acad Sci USA 2011. https://doi.org/10.1073/pnas.1117849108.

12. Fernet M, Ponette V, Deniaud-Alexandre E, et al. Poly (ADPRibrose, polymerase, a major determinant of early cell response $\mathrm{X}$ ionising radiation. Int J Radiat Biol. 2000;76:73-84. 
13. Abbott BG, Case JA, Dorbala S, Einstein AJ, Galt JR, Pagnanelli R, Bullock-Palmer RP, Soman P, Wells RG. Contemporary cardiac SPECT imaging-innovations and best practices: An information statement from the american society of nuclear cardiology. J Nucl Cardiol 2018;11:e000020.

14. Sharir T, Ben-Haim S, Merzon K, Prochorov V, Dickman D, BenHaim S, et al. High-speed myocardial perfusion imaging: Initial clinical comparison with conventional dual detector anger camera imaging. JACC Cardiovasc Imaging 2008;1:156-63.

15. Esteves FP, Raggi P, Folks RD, Keidar Z, Askew JW, Rispler S, et al. Novel solid-state-detector dedicated cardiac camera for fast myocardial perfusion imaging: Multicenter comparison with standard dual detector cameras. J Nucl Cardiol 2009;16:927-34.

16. Radiation Risk in Perspective: Position Statement of the Health Physics Society, http://hps.org/documents/risk_ps010-3.pdf, 2010, revised 2016, last accessed 19 June 2019.

17. National Council on Radiation Protection and Measurements. Uncertainties in fatal cancer risk estimates used in radiation protection. Bethesda, MD: National Council on Radiation Protection and Measurements; NCRP, Report No. 126; 1997.

18. Bateman TM, Heller GV, McGhie AI, Friedman JD, Case JA, Bryngelson JR, Hertenstein GK, Moutray KL, Reid K, Cullom SJ.
Diagnostic accuracy of rest/stress ECG-gated Rb-82 myocardial perfusion PET: Comparison with ECG-gated Tc-99m sestamibi SPECT. J Nucl Cardiol 2006;13:24-33.

19. Murthy VL, Bateman TM, Beanlands RS, Berman DS, BorgesNeto S, Chareonthaitawee P, Cerqueira MD, deKemp RA, DePuey EG, Dilsizian V, Dorbala S, Ficaro EP, Garcia EV, Gewirtz H, Heller GV, Lewin H, Mann A, Malhotra S, Ruddy TD, Schindler TH, Schwartz RG, Slomka PJ, Soman P, DiCarli MF. Clinical quantification of myocardial blood flow using positron emission tomography: A Joint Position Paper of the Cardiovascular Council (CVC) of the Society of Nuclear Medicine and Molecular Imaging (SNMMI) \& the American Society of Nuclear Cardiology (ASNC). J Nucl Cardiol 2018;25:269-97.

20. Wells RG, Marvin B, Poirier M, Renaud JM, deKemp RA, Ruddy TD. Optimization of SPECT measurement of myocardial blood flow with corrections for attenuation, motion, and blood-binding compared to PET. J Nucl Med 2017;58:2013-9. https://doi.org/10. 2967/jnumed.117.191049.

Publisher's Note Springer Nature remains neutral with regard to jurisdictional claims in published maps and institutional affiliations. 